\title{
Erratum to: Assessment of Blood Contamination in Biological Fluids Using MALDI-TOF MS
}

\author{
Katrina Laks ${ }^{1,2}$ - Tiina Kirsipuu ${ }^{1,2}$ - Tuuli Dmitrijeva ${ }^{1}$ Andres Salumets ${ }^{2,3}$. \\ Peep Palumaa ${ }^{1,2}$
}

Published online: 21 April 2016

(C) Springer Science+Business Media New York 2016

\section{Erratum to: Protein J}

DOI 10.1007/s10930-016-9657-y

The original version of this article unfortunately contained a mistake. The presentation of Fig. 2 was incorrect. The corrected Fig. 2 is given below.

The online version of the original article can be found under doi:10.1007/s10930-016-9657-y.

Katrina Laks

katrina.laks@gmail.com

1 Department of Gene Technology, Tallinn University of Technology, Akadeemia st 15, 12618 Tallinn, Estonia

2 Competence Centre on Health Technologies, Tiigi 61b, 50410 Tartu, Estonia

3 Institute of Bio- and Translational Medicine, University of Tartu, Ravila 19, 50411 Tartu, Estonia 


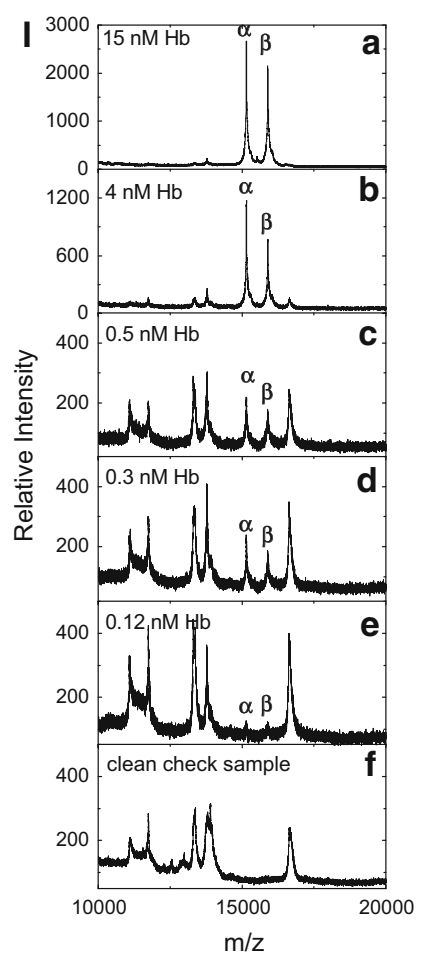



Fig. 2 Blood contamination detection in biological fluids. I MALDITOF MS spectra of CSF. a-e Artificially Hb-contaminated CSF. $\alpha$ and $\beta$ denote the $\mathrm{Hb} \alpha-$ and $\beta$-chains $(15.1$ and $15.9 \mathrm{kDa}$, respectively). f Negative control-uncontaminated CSF. II MALDITOF MS spectra of CSF. a-c Contaminated CSF samples.
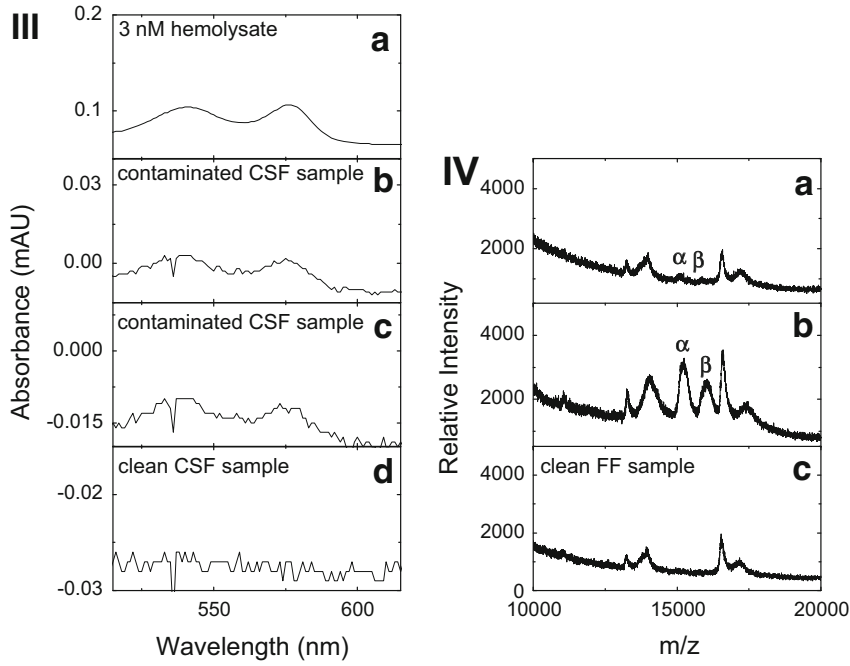

d Uncontaminated CSF sample. III Spectrophotometric analysis of CSF (515-615 nm). a The 3.0-nM hemolysate absorbance spectrum. b, c Contaminated CSF. d Uncontaminated CSF. IV MALDI-TOF MS spectra of FF. a, b Contaminated FF samples. c Uncontaminated FF sample 